\title{
The effect of intravenous infusion on the rapid recovery of elderly patients treated with painless colonoscopy and the value of ultrasonic measurement of the inferior vena cava diameter in guiding intravenous infusion
}

\author{
Qingqin Xu ${ }^{1,2}$, Hong Tu ${ }^{1}$, Shunju Xiang ${ }^{3,4}$, Qinghua Tan ${ }^{5}$, Xiao Wang ${ }^{1}$ \\ ${ }^{1}$ Department of Anesthesiology, West China Hospital of Sichuan University, Chengdu, China; ${ }^{2}$ Department of Anesthesiology, Chongqing General \\ Hospital, Chongqing, China; ${ }^{3}$ West China School of Nursing, Sichuan University, Chengdu, China; ${ }^{4}$ Department of Anesthesiology, West China \\ Hospital, Chengdu, China; ${ }^{5}$ Department of Gastroenterology, West China Hospital of Sichuan University, Chengdu, China \\ Contributions: (I) Conception and design: Q Xu, X Wang; (II) Administrative support: X Wang; (III) Provision of study materials or patients: Q Xu, Q \\ Tan; (IV) Collection and assembly of data: Q Xu, S Xiang; (V) Data analysis and interpretation: Q Xu, H Tu; (VI) Manuscript writing: All authors; (VII) \\ Final approval of manuscript: All authors. \\ Correspondence to: Xiao Wang. Department of Anesthesiology, West China Hospital of Sichuan University, Chengdu, China. \\ Email: 1490829116@qq.com.
}

Background Elderly patients with painless colonoscopy often complain listlessness, fatigue when they left postanesthesia care unit (PACU). These patients also commonly occur hypotension during anesthesia. However, intravenous infusion and blood volume assessment are seldom performed in elderly patients. Therefore, we aimed to observe the effect of intravenous infusion and to explore the value of inferior vena cava diameter (IVCD) measurement by ultrasound in rapid recovery.

Methods: Randomized, controlled, clinical trials, elderly patients (aged $\geq 60$ years) with painless colonoscopy were randomly divided into two groups: intravenous infusion versus no intravenous infusion was administered procedure. The primary outcome observed was the time required for complete recovery from anesthesia. Secondary outcomes included willingness to accept follow-up colonoscopy, discomfort symptoms, changes in the inferior vena cava (IVC)-related parameters. Hypotension was defined as a $\geq 20 \%$ decrease in systolic blood pressure (SBP) from baseline. The patients in the two groups were divided into nonhypotensive and hypotensive subgroups, respectively. Receiver operating characteristic (ROC) curves were used to determine the efficacy of the IVC-related parameters for predicting hypotension and the optimal threshold.

Results: A total of 65 patients were enrolled, including 34 patients in the infusion group and 31 patients in the conventional group. Administration of intravenous infusion reduced the time required for complete recovery and increased the willingness to accept follow-up colonoscopy and reduced discomfort symptoms. The IVC-related parameters were changed before and after bowel preparation and after reaching infusion loading dose. The ROC curve was used to predict hypotension during anesthesia. The maximum value of inferior vena cava diameter $\left(\mathrm{IVCD}_{\max }\right)$ was the best predictive efficacy in the both groups [area under the ROC curve $(\mathrm{AUC})=0.839$, $\mathrm{AUC}=0.877$, respectively $]$.

Conclusions: Intravenous infusion promotes the rapid recovery of elderly patients with painless colonoscopy. Measurement of the $\mathrm{IVCD}_{\max }$ can help to guide intravenous infusion.

Keywords: Inferior vena cava diameter (IVCD); elderly patients; painless colonoscopy; intravenous infusion; rapid recovery

Submitted Aug 19, 2020. Accepted for publication Jan 11, 2021.

doi: 10.21037/apm-20-2217

View this article at: http://dx.doi.org/10.21037/apm-20-2217 


\section{Introduction}

The risk of colon cancer rises with age. Goh et al.'s (1) study of an Asian population shows that more than 70\% of colorectal cancer patients are over the age of 65 years. Colonoscopy has high sensitivity for detecting colon cancer and precancerous lesions, however, colonoscopy is an invasive procedure and usually requires deep sedation. Elderly patients who undergo painless colonoscopy often complain of malaise, fatigue, when they left PACU. Moreover, elderly patients who have been fasting and bowel preparation are especially susceptible to developing hypotension (2). Intravenous infusion is rarely administered to elderly patients who receive painless colonoscopy in the clinical setting, and the benefits of intravenous infusion on rapid recovery after anesthesia have rarely been reported.

With the advancement of ultrasound technology, the American Society of Ultrasound and the Emergency Medicine Association specifically recommends the change in the IVCD during the respiratory cycle evaluation blood volume (3). The IVC is located in front of the thoracic 12 and lumbar 1 vertebral bodies. It has no venous valves and is a highly compliant blood vessel spanning the liver and diaphragm. During spontaneous breathing, the diameter of the IVC changes due to changes in intrathoracic pressure caused by inspiration and expiration (4). During inspiration, negative pressure in the chest cavity increases and increases venous return to the heart, causing the diameter of the IVC to reduce. During expiration, negative pressure in the chest cavity decreases and decreases venous return to the heart, resulting in an increase in the diameter of the IVC. Changes in the diameter of the IVC with spontaneous breathing are especially obvious during hypovolemia. However, while some studies $(5,6)$ have assessed the IVC to determine volume responsiveness, few studies have investigated the rapid assessment of blood volume in elderly patients undergoing painless colonoscopy using ultrasonic measurement of the IVC diameter (IVCD). Therefore, this study, enrolled elderly patients who would undergo painless colonoscopy, aimed to observe the effects of intravenous infusion and the application value of ultrasonic measurement of the IVCD in the rapid recovery of these patients.

We hypothesized that intravenous infusion was effective for elderly patients undergoing painless colonoscopy in the respect of rapid recovery and ultrasonic measurement of the IVCD was valuable for the assessment. We present the following article in accordance with the STARD reporting checklist (available at http://dx.doi.org/10.21037/apm-202217).

\section{Methods}

\section{General information}

This study is a single-center, sing-blind prospective randomized trial. All procedures performed in this study involving human participants were in accordance with the Declaration of Helsinki (as revised in 2013). This research was approved by the Medical Ethics Committee of West China Hospital of Sichuan University (No. 421, 2018) and was registered at http://www.chictr.org.cn (ChiCTR1800019595).

All patients or their guardians signed informed consent before the commencement of the study. A total of 65 elderly patients were selected for elective painless colonoscopy at the Center for Digestive Endoscopy of West China Hospital of Sichuan University between October 2018 and June 2019. There were 31 males and 34 females, ranging in age from $60-80$ years old. The patients all had a body mass index (BMI) in the range of $18-25 \mathrm{~kg} / \mathrm{m}^{2}$ and were American Society of Anesthesiologists (ASA) grade I-III. The exclusion criteria were: (I) had fasted for several days; (II) with arrhythmia or poor ultrasound image quality; (III) with moderate to massive regurgitation or stenosis of heart valves; (IV) with portal hypertension; (V) increased intra-abdominal pressure; (VI) with high risk of reflux aspiration; (VII) with ASA grade III who were in an unstable state after treatment; (VIII) had tracheal intubation under general anesthesia; (IX) had received intravenous infusion before ultrasound. The withdrawal criterion was the failure to complete bowl preparation as prescribed.

All patients were randomly divided into two groups by computer software. After bowel preparation, patients in the infusion group received intravenous crystal solution (compound sodium chloride). According to the 4-2-1 rehydration principle, the accumulated lost physiological fluid requirements were calculated based on the last fasting time. After the rapid infusion of one-half of the physiological fluid requirements as the load, painless colonoscopy was performed. Continuous infusion of the physical fluid requirements per hour was maintained until the patient left the PACU. Patients in the conventional group underwent painless colonoscopy after the bowel preparation without any liquid infusion (Figure 1). 


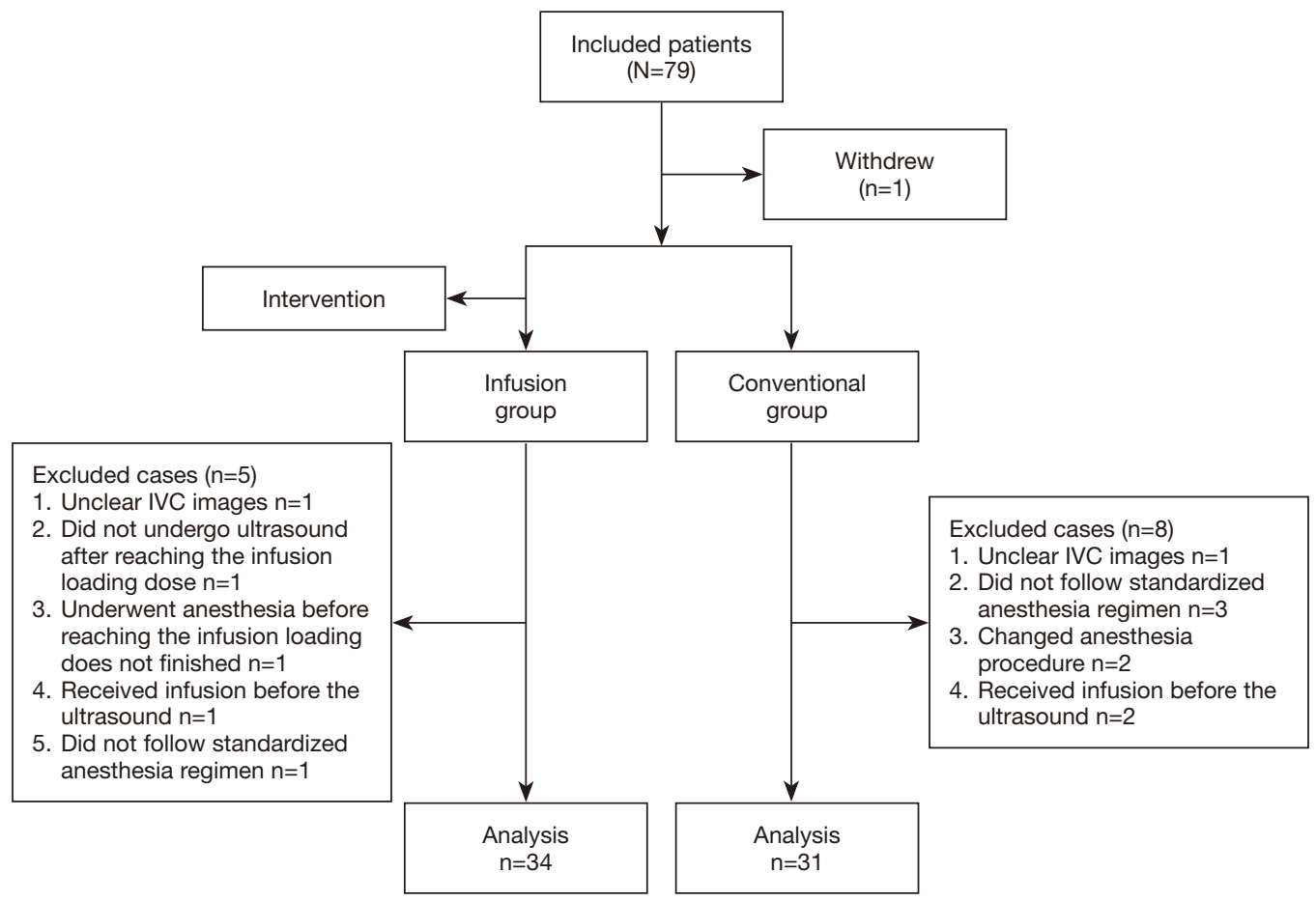

Figure 1 Process of patient screening, registration, and randomization.

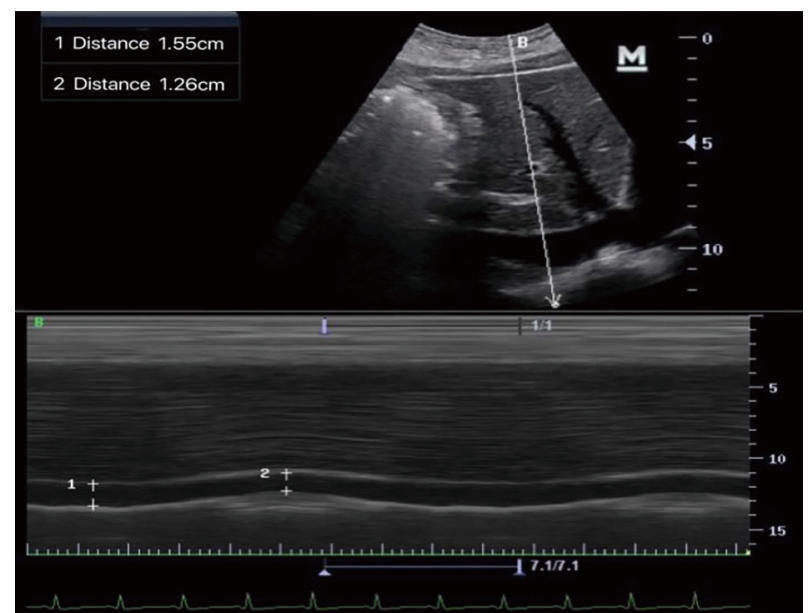

Figure 2 Schematic diagram of the inferior vena cava diameter (IVCD) in the long-axis view. Note: 1. IVCD $_{\max }$ : the maximum value of IVCD measured during expiration in a single respiratory cycle; 2 . IVCD $_{\min }$ : the minimum value of IVCD measured during inspiration in a single respiratory cycle.

\section{Ultrasonic measurement}

Patients were placed in the supine position. After at least 5 minutes of quiet breathing, ultrasonic measurement of the IVC was performed by a technician with 3 months of training. The longitudinal sections under the standard xiphoid process were usually measured by the Mindray M7 ultrasound diagnostic instrument and phased-array probe with a frequency of $2.5-3.5 \mathrm{MHz}$. The images showed the long axis of the IVC connecting to the right atrium. The $\mathrm{IVCD}_{\max }$ (it was defined as the maximum value measured when IVCD increased during expiration) and $\operatorname{IVCD}_{\text {min }}$ (it was defined as the minimum value measured when IVCD decrease during inspiration) were measured at the site $1-2 \mathrm{~cm}$ distal to the IVC-hepatic vein junction (Figure 2). The IVC was visualized and the images were frozen, and the distance between the inner edges of the IVC walls was measured by a scale in centimeters. The $\mathrm{IVCD}_{\max }$ and $\mathrm{IVCD}_{\min }$ were measured in three consecutive respiratory cycles, and the 
Table 1 Discharge standards after anesthesia

\begin{tabular}{|c|c|c|}
\hline Indicator & Score & Standard \\
\hline \multirow[t]{3}{*}{ Vital signs } & 2 & $\begin{array}{l}\text { Fluctuating within the } 20 \% \text { of preoperative } \\
\text { level }\end{array}$ \\
\hline & 1 & Between $20-40 \%$ of the preoperative level \\
\hline & 0 & $40 \%$ of the preoperative level \\
\hline \multirow[t]{3}{*}{ Walk } & 2 & $\begin{array}{l}\text { Able to determine orientation and steady } \\
\text { gait }\end{array}$ \\
\hline & 1 & $\begin{array}{l}\text { Able to determine orientation and/or steady } \\
\text { gait }\end{array}$ \\
\hline & 0 & Cannot meet any of above two items \\
\hline \multirow{3}{*}{$\begin{array}{l}\text { Nausea and } \\
\text { vomiting }\end{array}$} & 2 & Rare \\
\hline & 1 & Moderate \\
\hline & 0 & Severe \\
\hline \multirow[t]{3}{*}{ Pain } & 2 & Rare \\
\hline & 1 & Moderate \\
\hline & 0 & Severe \\
\hline \multirow{3}{*}{$\begin{array}{l}\text { Surgical } \\
\text { bleeding }\end{array}$} & 2 & Rare \\
\hline & 1 & Moderate \\
\hline & 0 & Severe \\
\hline
\end{tabular}

Note: 10 points is the highest score. Patients scoring $\geq 9$ points could be discharged.

mean values were calculated. The IVC collapse index (IVC-CI) was calculated according to the following equation: $\mathrm{IVC}-\mathrm{CI}=\left(\mathrm{IVCD}_{\max }-\mathrm{IVCD}_{\min }\right) / \mathrm{IVCD}_{\max } \times 100 \%$.

\section{Anesthesia}

The patients in both groups were administered general anesthesia after fasting for 10 hours. Intravenous access was established on the back of the right hand. The patients were placed in the left lateral position and the blood pressure cuff was placed on the left upper arm. Nasal cannula oxygen was given at $3 \mathrm{~L} / \mathrm{min}$. General anesthesia was induced with $0.1 \mu \mathrm{g} / \mathrm{kg}$ sufentanil and approximately $1-1.5 \mathrm{mg} / \mathrm{kg}$ propofol. The colonoscope was inserted when the patient reached Modified Observer's Assessment of Alertness/ Sedation score (MOAA/S) $\leq 1$ point (7) (patient showed no response to the call or no response to the mild flapping of the body) and no reflection of the eyelashes. If body movement, eyebrow furrowing, blinking, or coughing was observed, an additional $0.3 \mathrm{mg} / \mathrm{kg}$ propofol was injected intravenously to maintain the anesthesia.

Hypotension was defined as a reduction in systolic blood pressure (SBP) of more than $20 \%$ from baseline. After the completion of the colonoscopy, the patient was transferred to the PACU to recover. When the Steward recovery score was $\geq 4$ points, patients could leave the PACU (8). Patients were followed up after leaving PACU until they had made a complete recovery from anesthesia, which was indicated by the patients meeting the two following criteria: (I) a score of $\geq 9$ points on the Modified Post-Anesthetic Discharge Scoring System scale (MPADSS) (9) (Table 1); and (II) recovery to their pre-bowel-preparation mental state.

\section{Observation indicators}

Data collection was planned before the study were performed. The sex, age, BMI, duration of anesthesia, anesthetic drugs, baseline blood pressure (BP), and heart rate (HR) were collected for the two groups of patients. The time required for complete recovery from anesthesia, defined as the time interval between leaving the PACU and meeting the two criteria for complete recovery after anesthesia, was also recorded. When leaving the PACU, patients were asked about their degree of acceptance, which was defined as their willingness to accept follow-up colonoscopy, as well as whether they had any uncomfortable symptoms, such as thirst, nausea, vomiting, drowsiness, anxiety, starvation, fatigue, and dizziness. The $\mathrm{IVCD}_{\max }$, IVCDmin, SBP, diastolic blood pressure (DBP), mean arterial blood pressure (MBP), and HR of the two groups of patients were recorded before and after bowel preparation and after reaching the intravenous infusion loading dose, The IVC-CI was also calculated. The BP values during anesthesia were recorded. Hypotension was defined as a reduction of $\geq 20 \%$ in SBP from baseline; the baseline value of $\mathrm{BP}$ was defined as the BP before bowel preparation.

\section{Statistical analysis}

SPSS 22.0 (SPSS Inc. Released 2014. SPSS Statistics for Windows, Version 22.0. Chicago: SPSS Inc.) was used for statistical analysis. Quantitative data are described as the mean \pm standard deviation. Inter-group comparisons were performed using the $t$-test. Within-group comparisons were conducted using paired $t$-tests. The Wilcoxon ranksum test was used for inter-group comparisons of variables with non-normal distribution. Repeated-measures analysis of variance (ANOVA) was used to compare the means of 
Table 2 General information of patients in both groups $(\bar{x} \pm s)$

\begin{tabular}{lccc}
\hline Basic information & Infusion group $(\mathrm{n}=34)$ & Conventional group $(\mathrm{n}=31)$ & $\mathrm{P}$ \\
\hline Sex (male/female, $\mathrm{n})$ & $17 / 17$ & $14 / 17$ & 0.887 \\
Age (years) & $71.41 \pm 7.27$ & $71.61 \pm 7.77$ & 0.914 \\
BMI (kg/m $\left.{ }^{2}\right)$ & $22.63 \pm 3.64$ & $23.00 \pm 3.06$ & 0.663 \\
ASA (I/II/IIl, $\mathrm{n})$ & $4 / 17 / 13$ & $1 / 11 / 19$ & 0.130 \\
Duration of anesthesia (min) & $30.01 \pm 16.27$ & $26.91 \pm 16.99$ & 0.456 \\
Sufentanil (ug) & $6.53 \pm 1.48$ & $6.23 \pm 1.20$ & 0.387 \\
Propofol (mg) & $168.82 \pm 89.49$ & $129.35 \pm 81.12$ & 0.068 \\
SBP (mmHg) & $130.09 \pm 15.24$ & $136.94 \pm 15.31$ & 0.076 \\
DBP (mmHg) & $74.15 \pm 12.74$ & $78.61 \pm 10.28$ & 0.127 \\
MBP (mmHg) & $92.79 \pm 11.88$ & $98.05 \pm 9.83$ & 0.058 \\
HR (times/min) & $77.03 \pm 16.75$ & $83.52 \pm 12.57$ & 0.085 \\
\hline
\end{tabular}

$\mathrm{BMI}$, body mass index [= body weight $(\mathrm{kg}) /$ height $\left.^{2}(\mathrm{~m})\right]$; ASA, American Society of Anesthesia; SBP, systolic blood pressure; DBP, diastolic blood pressure; MBP, mean arterial pressure; HR, heart rate.

the quantitative data based on repeated measurements, and the least significant difference method was used for pairwise comparisons. The chi-squared test was performed to compare inter-group differences of qualitative data, and the paired chi-squared test was used to compare the intragroup differences before and after bowel preparation. The count data are described as frequencies (percentages). Correlations between IVC parameters and hypotension were calculated using Pearson's correlation analysis. The best indicator for predicting intraoperative hypotension was determined according to the value of the area under the ROC curve (AUC), and the best diagnostic threshold was selected at the point where the sum of sensitivity and specificity was greatest in the ROC. AUCs were compared with the Delong test. For all statistical analyses, $\mathrm{P}<0.05$ was considered statistically significant.

The sample size was calculated based on the time point of complete recovery after anesthesia, and the time was used as the main indicator of the outcome. According to the preliminary experiment, the difference between the times of the two groups was assumed to be 0.5 hours. The probability of a type I error was set at 0.05 , and the probability of a type II error was set at 0.1 . Besides, the sample size of the two groups was assumed to be the same. The calculation results showed that to achieve $90 \%$ power in a test, a total of 36 cases were required. Considering a dropout rate of $10 \%$, the final sample size was 40 cases. The following formula was used:

$$
n_{1}=n_{2}=2\left(\frac{\left(t_{\alpha}+t_{\beta}\right)^{2} s}{\delta}\right)^{2}
$$

where $\delta$ is the difference in the time required for complete recovery between the two groups in the preliminary experiment; $t_{\alpha}$ and $t_{\beta}$ correspond to the quantiles of type 1 and type 2 errors, respectively; and $\mathrm{s}$ is the standard deviation of the full recovery time in the preliminary experiment.

\section{Results}

\section{General information}

A total of 79 patients were initially enrolled. Of them, 14 patients were excluded from the analysis (Figure 1), and 65 patients were eventually included and randomly divided into two groups. There were 34 and 31 patients in the infusion group and the conventional group, respectively. There were no statistically significant differences found in sex, age, BMI, ASA grade, duration of anesthesia, total amount of anesthetic drugs, SBP, DBP, MBP, or HR between the two groups $(\mathrm{P}>0.05$, Table 2$)$.

\section{Comparison of the time required for complete recovery from anesthesia, willingness accept to follow-up colonoscopy, incidence of discomfort symptoms in the two groups}

The time required for complete recovery from anesthesia 
Table 3 Comparison of time required for recovery from anesthesia between the two groups $(\bar{x} \pm s)$

\begin{tabular}{lcc}
\hline Variable & Infusion group $(\mathrm{n}=34)$ & Conventional group $(\mathrm{n}=31)$ \\
\hline Time required for complete recovery from anesthesia $(\mathrm{h})$ & $0.97 \pm 0.91$ & $2.25 \pm 2.08$ \\
\hline Indicators are expressed as mean \pm standard deviation. & & 0.002 \\
\hline
\end{tabular}

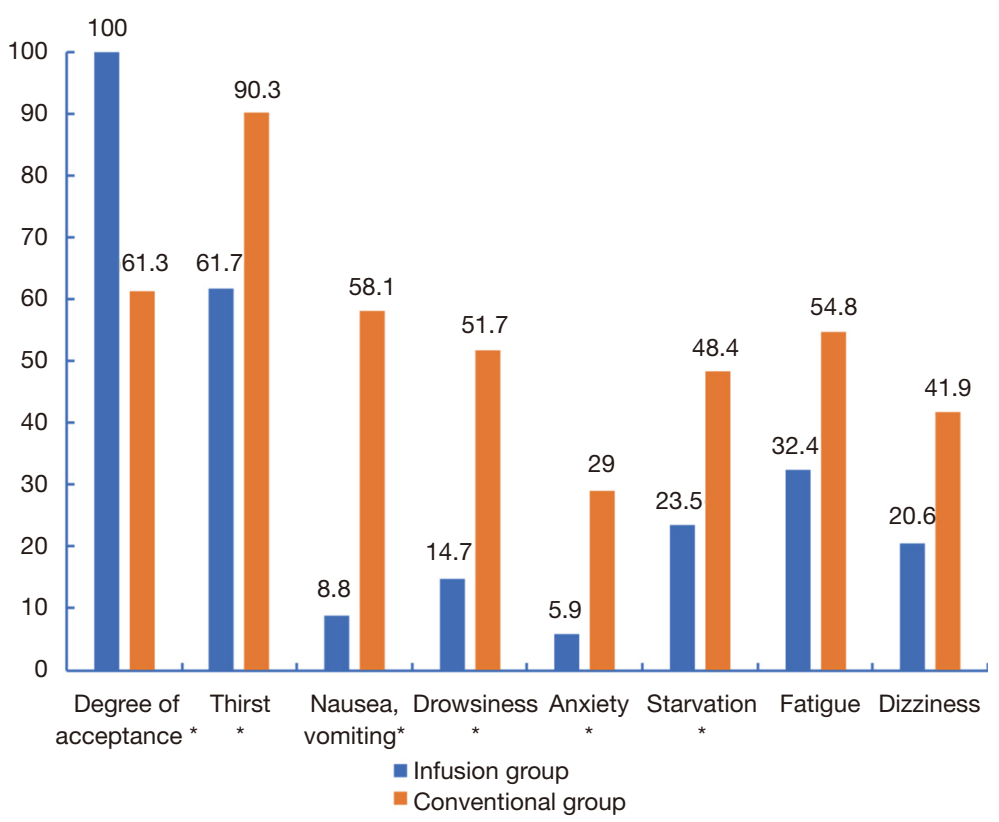

Figure 3 Percentage of patients with follow-up acceptance and discomfort symptoms in both groups. Note: * indicates compared with conventional group $\mathrm{P}<0.05$.

was significantly shorter in the infusion group $(\mathrm{P}=0.002$, Table 3). There was a significant difference in the willingness of the two groups to accept to follow-up colonoscopy $(\mathrm{P}<0.001)$. The incidence of discomfort symptoms was significantly reduced in the infusion group $(\mathrm{P}<0.001$, $\mathrm{P}<0.001, \mathrm{P}=0.001, \mathrm{P}=0.012, \mathrm{P}=0.032, \mathrm{P}=0.055, \mathrm{P}=0.083$, Figure 3).

\section{Comparison of IVCD $D_{\max }, I V C D_{\text {min }}, I V C-C I, S B P, D B P$, $M B P$ in the before and after bowel preparation and after intravenous infusion of load in the two groups}

After bowel preparation, the IVCD ${ }_{\max }$ and $\mathrm{IVCD}_{\min }$ of the two groups were significantly smaller than those before bowel preparation, while the IVC-CI was significantly higher than that before bowel preparation (all $\mathrm{P}<0.001$, Figure $4 A, B)$. In the infusion group, the $\operatorname{IVCD}_{\max }$ and $I V C D_{\min }$ were significantly larger after reaching the infusion load than they were after bowel preparation
$(\mathrm{P}<0.001, \mathrm{P}<0.001)$, and the IVC-CI was significantly lower than it was after the bowel preparation $(\mathrm{P}<0.001$, Figure $4 A)$. No significant change was observed in $\mathrm{BP}$ between the two groups before and after bowel preparation or after intravenous infusion of load $(\mathrm{P}>0.05$, Table 4).

\section{Comparison of reduction of SBP, DBP and MBP in the bypotensive subgroups of the two groups}

No significant difference was found in the reductions of SBP, DBP, and MBP during anesthesia in the hypotensive subgroups of the two groups $(\mathrm{P}=0.202, \mathrm{P}=0.530, \mathrm{P}=0.420$, Table 5).

Comparison of characteristics, baseline blood pressure, and IVC-related parameters before anesthesia in the nonbypotensive and hypotensive subgroups

According to the definition of hypotension, the infusion 

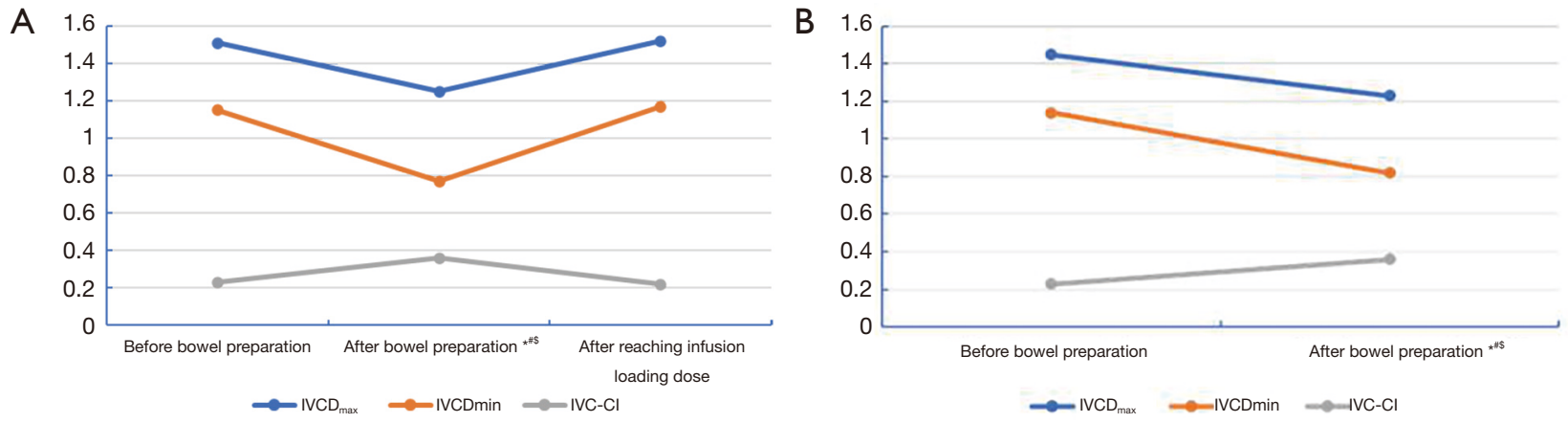

Figure 4 Inferior vena cava-related parameters measured at each time point in both groups. (A) Inferior vena cava-related parameters measured at various time points in the infusion group. Note: * indicates $\mathrm{IVCD}_{\max }$ compared with that of before bowel preparation $\mathrm{P}<0.05$; ${ }^{*}$ indicates $\mathrm{IVCD}_{\min }$ compared with that before bowel preparation $\mathrm{P}<0.05 ;{ }^{\$}$ indicates IVC-CI compared with that before bowel preparation $\mathrm{P}<0.05$. (B) Inferior vena cava-related parameters measured at various time points in the conventional group. Note: * indicates $\mathrm{IVCD}_{\max }$ compared with that before bowel preparation $\mathrm{P}<0.05$; $^{\#}$ indicates $\mathrm{IVCD}_{\min }$ compared with that before bowel preparation $\mathrm{P}<0.05$; ${ }^{\$}$ indicates IVC-CI compared with that before bowel preparation $\mathrm{P}<0.05$. IVCD, inferior vena cava diameter; IVC-CI, inferior vena cava collapse index.

Table 4 Comparison of inferior vena cava-related parameters and blood pressure between the two groups before and after bowel preparation and after reaching infusion loading dose

\begin{tabular}{|c|c|c|c|c|c|c|c|c|c|}
\hline Indicator & \multicolumn{5}{|c|}{ Infusion group } & \multicolumn{4}{|c|}{ Conventional group } \\
\hline $\mathrm{IVCD}_{\max }(\mathrm{cm})$ & $1.51 \pm 0.39$ & $1.25 \pm 0.37^{\star}$ & $1.52 \pm 0.31$ & 14.271 & $<0.001$ & $1.45 \pm 0.40$ & $1.23 \pm 0.47$ & 3.28 & $<0.001$ \\
\hline $\mathrm{IVCD}_{\min }(\mathrm{cm})$ & $1.15 \pm 0.34$ & $0.77 \pm 0.27^{\star}$ & $1.17 \pm 0.28$ & 27.261 & $<0.001$ & $1.14 \pm 0.43$ & $0.82 \pm 0.45$ & 4.49 & $<0.001$ \\
\hline $\mathrm{SBP}(\mathrm{mmHg})$ & $130.09 \pm 15.24$ & $129.53 \pm 17.82$ & $129.09 \pm 15.29$ & 0.277 & 0.759 & $136.94 \pm 15.31$ & $140.52 \pm 19.32$ & -1.01 & 0.32 \\
\hline $\mathrm{DBP}(\mathrm{mmHg})$ & $74.15 \pm 12.74$ & $75.56 \pm 11.22$ & $75.03 \pm 8.50$ & 1.409 & 0.249 & $78.61 \pm 10.28$ & $80.68 \pm 11.56$ & -0.93 & 0.36 \\
\hline MBP $(\mathrm{mmHg})$ & $92.79 \pm 11.88$ & $93.68 \pm 12.51$ & $93.05 \pm 9.00$ & 1.407 & 0.249 & $98.05 \pm 9.83$ & $101.51 \pm 11.06$ & -1.45 & 0.16 \\
\hline
\end{tabular}

All parameters are presented as mean \pm standard deviation. *, indicates compared to that before bowel preparation $\mathrm{P}<0.001$. IVCD, inferior vena cava diameter; IVC-Cl, inferior vena cava collapse index; SBP, systolic blood pressure; DBP, diastolic blood pressure; MBP, mean arterial pressure.

Table 5 Blood pressure reduction in patients with hypotension during general anesthesia

\begin{tabular}{|c|c|c|c|}
\hline Indicator & Infusion group $(n=15)$ & Conventional group $(n=12)$ & $\mathrm{P}$ \\
\hline DBP reduction (\%) & $30.79 \pm 7.47$ & $33.51 \pm 13.29$ & 0.530 \\
\hline MBP reduction (\%) & $31.24 \pm 6.06$ & $34.02 \pm 9.91$ & 0.420 \\
\hline
\end{tabular}

All indicators are expressed as mean \pm standard deviation. SBP, systolic blood pressure; DBP, diastolic blood pressure; MBP, mean arterial pressure. 
Table 6 Comparison of characteristics, baseline blood pressure, and inferior vena cava parameters before anesthesia in the non-hypotensive and hypotensive subgroups

\begin{tabular}{|c|c|c|c|c|c|c|}
\hline Indicator & \multicolumn{3}{|c|}{ Infusion group } & \multicolumn{3}{|c|}{ Conventional group } \\
\hline ASA (I/II/III) & $3 / 9 / 7$ & $1 / 8 / 6$ & 0.714 & $0 / 9 / 10$ & $1 / 2 / 9$ & 0.126 \\
\hline Age (years) & $70.63 \pm 7.12$ & $72.40 \pm 7.58$ & 0.489 & $70.37 \pm 8.23$ & $73.58 \pm 6.86$ & 0.269 \\
\hline Propofol (mg) & $171.84 \pm 82.20$ & $165.00 \pm 100.82$ & 0.829 & $133.95 \pm 83.87$ & $122.08 \pm 79.64$ & 0.699 \\
\hline $\mathrm{DBP}(\mathrm{mmHg})$ & $73.89 \pm 15.84$ & $74.47 \pm 7.72$ & 0.899 & $78.89 \pm 9.92$ & $78.17 \pm 11.26$ & 0.851 \\
\hline $\mathrm{MBP}(\mathrm{mmHg})$ & $92.14 \pm 14.27$ & $93.62 \pm 8.32$ & 0.724 & $98.26 \pm 10.80$ & $97.72 \pm 11.26$ & 0.884 \\
\hline $\mathrm{IVCD}_{\max }(\mathrm{cm})$ & $1.67 \pm 0.25$ & $1.32 \pm 0.27$ & $<0.001$ & $1.47 \pm 0.40$ & $0.86 \pm 0.31$ & $<0.001$ \\
\hline $\mathrm{IVCD}_{\min }(\mathrm{cm})$ & $1.24 \pm 0.26$ & $1.09 \pm 0.29$ & 0.131 & $1.03 \pm 0.45$ & $0.50 \pm 0.20$ & 0.001 \\
\hline
\end{tabular}

All indicators are expressed as mean \pm standard deviation. ASA, American Society of Anesthesia Classification; SBP, systolic blood pressure; DBP, diastolic blood pressure; MBP, mean arterial pressure; IVCD, inferior vena cava diameter; IVC-CI, inferior vena cava collapse index.

Table 7 Pearson correlation between each inferior vena cava-related parameters and the presence of hypotension

\begin{tabular}{lcccc}
\hline \multirow{2}{*}{ Parameter } & \multicolumn{2}{c}{ Infusion group $(\mathrm{n}=15)$} & \multicolumn{2}{c}{ Conventional group $(\mathrm{n}=12)$} \\
\cline { 2 - 5 } & $\mathrm{r}$ & $\mathrm{P}$ & $\mathrm{r}$ & $<$ \\
\hline $\mathrm{IVCD}_{\max }$ & -0.572 & $<0.001$ & -0.637 & $<0.001$ \\
$\mathrm{IVCD}_{\min }$ & -0.264 & 0.131 & -0.577 & $<0.001$ \\
$\mathrm{IVC}-\mathrm{Cl}$ & -0.278 & 0.123 & 0.293 & 0.123 \\
\hline
\end{tabular}

IVCD, inferior vena cava diameter; IVC-CI, inferior vena cava collapse index.

group and the conventional group were divided into nonhypotensive and hypotensive subgroups, respectively. There was no significant difference found in terms of ASA grade, age, total amount of propofol, or baseline blood pressure between the two subgroups in either group (all $\mathrm{P}>0.05)$. The $\mathrm{IVCD}_{\max }$ of the patients with hypotension was significantly lower than that of patients without hypotension $(\mathrm{P}<0.001, \mathrm{P}<0.001$, Table 6).

\section{Pearson correlation between each IVC-related parameters and the presence of hypotension}

Pearson's correlation analysis showed that the correlation coefficients of the $\mathrm{IVCD}_{\max }$ and $\mathrm{IVCD}_{\min }$ in the hypotensive subgroups in the infusion group and the conventional group were $r=-0.572$ and $r=-0.264$, and $r=-0.637$ and $r=-0.577$, respectively. The correlation coefficient between the IVC-
$\mathrm{CI}$ and hypotension in the infusion group was $(\mathrm{r}=-0.278)$, and the correlation coefficient between the IVC-CI and hypotension in the conventional group was $\mathrm{r}=0.293$ (Table 7).

\section{ROC analysis of various IVC-related parameters in predicting bypotension}

The efficacy of IVC-related parameters in predicting hypotension was examined by ROC curve analysis. In the infusion group, the results of ROC curve analysis showed that the AUC values of the IVCD ${ }_{\text {max }}$ and $I V C D_{\min }$ were $0.839(0.69,0.99)$ and $0.739(0.56,0.92)$, respectively, which showed IVCD ${ }_{\text {max }}$ to have statistically significantly better predictive efficacy than $\mathrm{IVCD}_{\text {min }}(\mathrm{P}=0.006)$. The difference between the AUC values of IVC-CI and IVCD ${ }_{\max }$ was not statistically significant $(\mathrm{P}=0.262)$. The difference between the AUC values of VVCD $_{\text {min }}$ and IVC-CI was also not statistically 
Table 8 ROC analysis of various inferior vena cava parameters in predicting hypotension

\begin{tabular}{|c|c|c|c|c|c|c|}
\hline Parameter & \multicolumn{3}{|c|}{ Infusion group } & \multicolumn{3}{|c|}{ Conventional group } \\
\hline Optimal threshold value & 1.25 & 0.78 & 0.39 & 1.23 & 0.82 & 0.37 \\
\hline Sensitivity & 0.87 & 0.73 & 0.67 & 1 & 1 & 0.82 \\
\hline Specificity & 0.84 & 0.79 & 0.59 & 0.79 & 0.68 & 0.61 \\
\hline $95 \%$ confidence interval & $(0.69,0.99)$ & $(0.56,0.92)$ & $(0.37,0.78)$ & $(0.75,1)$ & $(0.73,0.99)$ & $(0.48,0.88)$ \\
\hline
\end{tabular}

*, indicates compared with the IVCD $\mathrm{max}_{\text {of }}$ the same group, $\mathrm{P}<0.05$. ROC, receiver operating characteristic; IVCD, inferior vena cava diameter; IVC-Cl, inferior vena cava collapse index; AUC, area under the ROC curve.
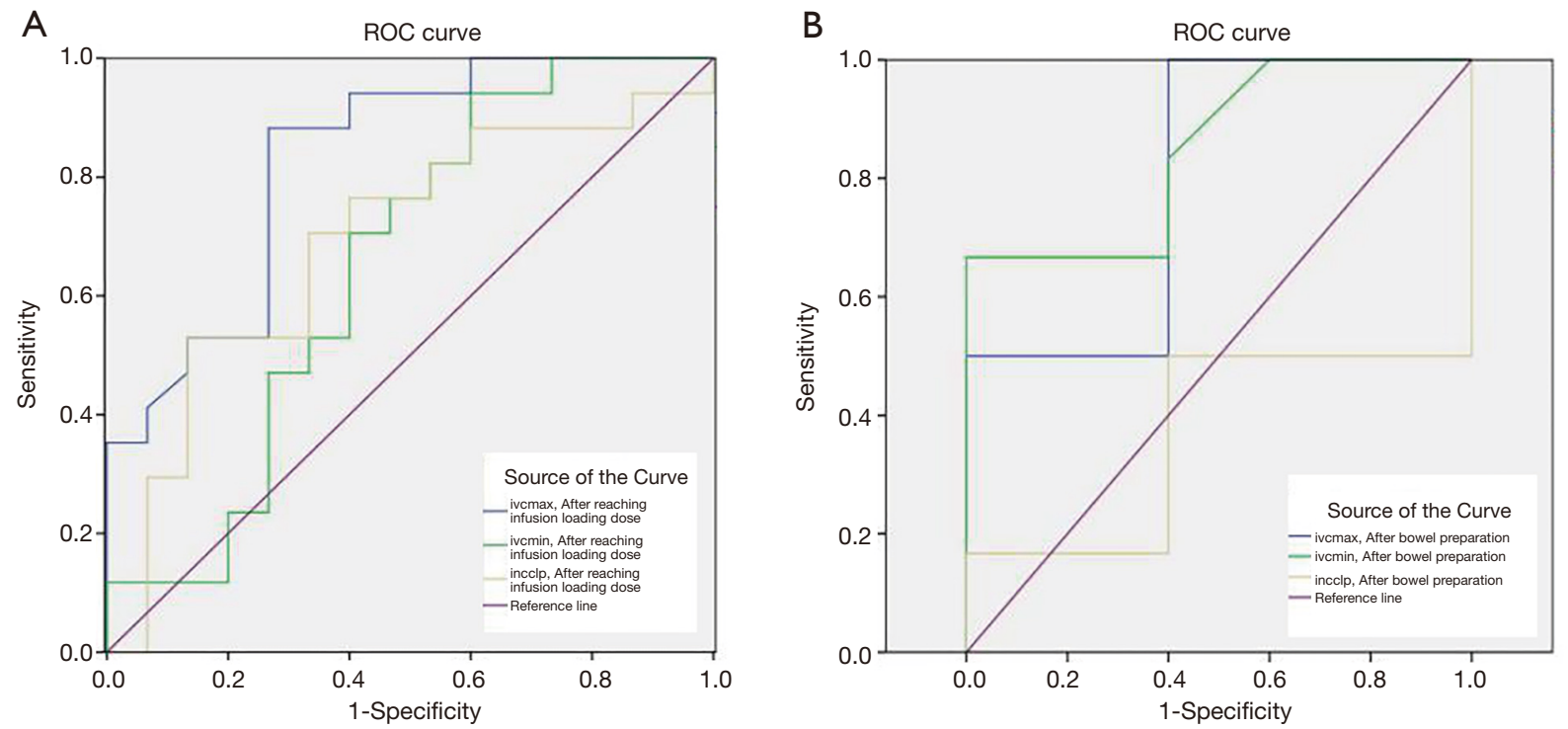

Figure 5 ROC curves of inferior vena cava-related parameters in both groups. ROC curves of inferior vena cava-related parameters in (A) the infusion group; (B) the conventional group. ROC, receiver operating characteristic.

significant $(\mathrm{P}=0.824)$. The optimal threshold value of the IVCD $_{\max }$ was $1.25 \mathrm{~cm}$, which had a sensitivity of $87 \%$ $(0.7,1.04)$ and a specificity of $84 \%(0.675,1.005)$ (Table 8 and Figure $5 A$ ). In the conventional group, the results of ROC curve analysis showed that the AUC values of the $\mathrm{IVCD}_{\max }$ and $\mathrm{IVCD}_{\min }$ were $0.877(0.75,1)$ and $0.86(0.73$, 0.99), respectively. The AUC value of $\mathrm{IVCD}_{\max }$ showed it to have statistically significantly better predictive efficacy than IVC-CI $(\mathrm{P}=0.009)$. However, the difference between the AUC values of IVCD $_{\min }$ and IVC-CI was not statistically significant $(\mathrm{P}=0.053)$, nor was the difference in the AUC values of $\operatorname{IVCD}_{\max }$ and $\operatorname{IVCD}_{\min }(\mathrm{P}=0.661)$. The optimal threshold value of the $\mathrm{IVCD}_{\max }$ was $1.23 \mathrm{~cm}$, which had a sensitivity of $100 \%(1,1)$ and a specificity of $79 \%(0.607$, 0.973) (Table 8 and Figure 5B).

\section{Discussion}

Fasting and bowel preparation are indispensable steps in painless colonoscopy; however, they are also the main causes of dehydration. The issue of rehydration can be easily ignored due to the interventional procedure of digestive endoscopy, which makes endoscopy more likely to aggravate the degree of dehydration. Long-term dehydration not only causes discomfort symptoms such as thirst, starvation, anxiety, nausea, and vomiting, but it also increases the 
incidence of postoperative nausea and vomiting (2), which can affect patients' rapid recovery. Therefore, the effects of intravenous infusion versus no intravenous infusion on rapid recovery in elderly patients undergoing painless colonoscopy were investigated.

A total of 65 patients were included for analysis in this study (Figure 1). There were no statistical different in general information of patients in both groups (Table 2). The primary outcome observed was the recovery time from anesthesia. Compared with the conventional group, the infusion group took a significantly shorter time to completely recover from anesthesia $(\mathrm{P}=0.002$, Table 3). According to the definition of complete recovery from anesthesia, a modified postanesthetic discharge scoring system scale was adopted for the follow-up after the patients had left the PACU (9) (Table 1). However, the scoring criteria, which evaluated vital signs, walking status, nausea, and vomiting, did not assess patients' mental state. Therefore, in addition to the scoring criteria, the patients' mental state was continually observed to ensure that they achieved complete recovery from anesthesia. The factors affecting the time of recovery from anesthesia may be as follows. Firstly, discomfort symptoms such as thirst, dizziness, and drowsiness are independent factors that prolong the time required to recover postoperatively $(10,11)$. Secondly, bowel preparation may lead to electrolyte disturbance (12). Hypocalcemia, hypokalemia, and other symptoms can cause general fatigue, apathy, and muscle tone decline. These symptoms negatively affect patients' mental states, thereby prolonging the time for complete recovery from anesthesia. Thirdly, kidney function decreases with age, and bowel preparation causes dehydration, which results in further damage to renal function (13), thus delaying the excretion of anesthetic drugs.

In order to solve the problem of rapid recovery from anesthesia, in the infusion group adopted two intravenous infusion methods: rapid intravenous infusion and maintenance intravenous infusion. Rapid intravenous infusion method was that, physiological fluid loss was calculated based on body weight and fasting time and half of the physiological fluid lost (approximately $500 \mathrm{~mL}$ ) was rapidly supplied via intravenous infusion, which was completed in about 30 minutes. Maintenance intravenous infusion method to reduce the perioperative loss of physiological fluid, the amount of physiological fluid lost per hour continued to be supplemented via intravenous infusion during the surgical period and in the PACU. The average infusion volume when the patients left the PACU was $800 \mathrm{~mL}$.

Preoperative fasting or bowel preparation caused physiological body fluid loss which is mainly composed of extracellular fluids and a small amount of electrolytes. Crystal fluid is often used to supplement the loss of physiological body fluid in the clinical setting (14). In addition to price cheap, compound sodium chloride injection contains sodium chloride and other electrolyte components. Intravenous fluid not only replenishes blood volume and maintains the balance of water and electrolytes, but it also promotes the excretion of anesthetic drugs. Therefore, intravenous infusion shortened the time to complete recovery from anesthesia in elderly patients who were treated with painless colonoscopy in this study.

A meta-study (15) suggested that dehydration symptoms was occurred commonly in minor operations these include thirst, drowsiness and dizziness. The administration of $500-1,000 \mathrm{~mL}$ of intravenous fluid was shown to improve postoperative thirst, but it had no significant effect on nausea, vomiting, drowsiness, or dizziness. However, when a higher amount of intravenous fluid $(>1,000 \mathrm{~mL})$ was administered, postoperative drowsiness, thirst, dizziness, nausea, and subjective well-being were reportedly improved. The secondary outcomes of this study included the willingness to accept follow-up colonoscopy and the incidence of discomfort symptoms. Figure 4 shows that compared with the conventional group, willingness to accept follow-up colonoscopy was significantly increased in the infusion group $(\mathrm{P}<0.001)$, while the incidence of discomfort symptoms (thirst, nausea, vomiting, drowsiness, anxiety, and starvation) was significantly reduced (all $\mathrm{P}<0.05)$. In this study, the administration of average 800 $\mathrm{mL}$ of intravenous fluids could, to a certain extent, improve both the acceptance rate of follow-up colonoscopy and dehydration symptoms. Our results regarding the incidence of discomfort symptoms, such as thirst and mood changes, were similar to those of Holte et al. (15).

Preoperative fasting and bowel preparation are considered to be causes of dehydration (6), which results in hypovolemia. However, in the present day, there are many indicators to assess blood volume in clinic, including stroke volume variation, pulse pressure variation, and central venous pressure (16). The acquisition of these indicators not only requires special monitoring equipment, but also involves invasive procedures, which limits the application of these indicators outside the operating room. There are some noninvasive methods to assess blood volume (17), such as passive legs raising test (PLRT), end-expiratory occlusion (EEO). Both of the two methods are reversible and temporary volume load. PLRT is suitable for patients with spontaneous breathing, but need special position and 
raise the lower extremities. It is not convenient to clinical operation. EEO is suitable for patients with mechanical ventilation. The secondary outcomes of this study included the use of ultrasound to measure the IVCD changes before and after bowel preparation and after reaching the infusion loading dose in all patients. Ultrasound showed that the $I V C D_{\text {max }}$ and IVCD ${ }_{\text {min }}$ were reduced, and the IVC-CI was increased after bowel preparation in both groups, while BP and HR showed no significant changes (Table 4, Figure 4). After $500 \mathrm{~mL}$ of intravenous infusion in the infusion group, ultrasound was immediately performed to measure the IVCD. Compared with the same group after bowel preparation, the $I V C D_{\text {max }}$ and $I V C D_{\text {min }}$ were increased by approximately 2.7 and $4 \mathrm{~mm}$, respectively, and the IVC-CI was decreased by $14 \%$. These observations reflected the close relationship between changes in IVCD and blood volume, which is in agreement with the results of other studies $(5,18-20)$. Because the IVC has no valve and can easily expand, its diameter varies with changes in blood volume. When the blood volume is insufficient, the IVC is not affected by the body's compensation (4). Therefore, in the infusion group, the diameter of IVC changed immediately after reaching the infusion loading dose, but the $\mathrm{BP}$ and $\mathrm{HR}$ did not change significantly, which was similar to the findings of Wo et al. (21). In the clinical setting, noninvasive BP and HR measurements are often used as basic hemodynamic monitoring indicators for painless colonoscopy patients; however, these indicators do not early identify hypovolemia and volume reaction (22). Ultrasonic measurement of IVCD is a non-time-consuming, simple, and repeatable procedure. Therefore, it can be used as a noninvasive and reliable tool for rapid assessment of blood volume in elderly patients who undergo painless colonoscopy.

Although the IVCD measured by ultrasound (Table 4) confirmed that the bowel preparation could cause hypovolemia in elderly patients, the changes in IVCrelated parameters did not accurately delineate the value of lost blood volume. When patients in the infusion group were given a rapid intravenous infusion of about $500 \mathrm{~mL}$, the IVCD $_{\max }$ expanded by more than $15 \%$, indicating that it was volume responsive and was on the ascending branch of the Frank-Staling curve. This observation showed that the volume load was effective (22). During anesthesia, there are often varying degrees of hypotension. Usually, SBP below $90 \mathrm{mmHg}$, baseline BP lower by $20 \%$ or $30 \%$, or MBP less than $50 \mathrm{mmHg}$ is the standard for hypotension (23). However, there is still no uniform definition of hypotension. Considering that the included population is elderly patients who often have cardiovascular and cerebrovascular diseases, it is necessary to maintain relatively higher BP levels perioperatively in order to ensure adequate perfusion of the vital organs with decreased function. Therefore, in this study, hypotension was defined as a reduction in SBP $\geq 20 \%$ relative to baseline for longer than 2 minutes. In this study, it was not completely clear whether volume load could effectively prevent hypotension during anesthesia. The degree of BP reduction was also one of the secondary outcomes. Hypotension occurred during anesthesia in both groups; 15 cases occurred in infusion group (SBP decreased by $30 \%$ ), and 12 cases occurred in the conventional group (SBP decreased by $34 \%$ ), and there was no statistically significant difference in the degree of BP decrease in the hypotensive subgroups in the two groups (Table 5). One reason for this result was that the long fasting period and bowel preparation led to massive body fluid loss, however, intravenous infusion $(500 \mathrm{~mL})$ could not fully and effectively prevent hypotension during anesthesia. Another possible reason is that larger intravenous infusion loads can effectively prevent hypotension, but this needs to be further investigated by expanding the sample size.

Hypotension during anesthesia is associated with factors such as ASA grade, age, baseline BP, and use of propofol (24). In this study, the infusion and conventional groups were further divided into non-hypotensive and hypotensive subgroups, and the factors that might affect blood pressure were analyzed. It was found that the ASA grade, age, baseline BP, and total amount of propofol administered showed no statistically significant difference between the nonhypotensive and hypotensive subgroups in either group. However, the $\mathrm{IVCD}_{\max }$ of patients in hypotensive subgroup was significantly smaller than that of the non-hypotensive group $(\mathrm{P}<0.001$, Table 6). Table 7 shows that hypotension and the IVCD ${ }_{\max }$ had maximal relevancy. Therefore, we postulated that the most common cause of hypotension in this study was related to blood volume during anesthesia.

Rapid intravenous infusion was designed to rapidly replenish the blood volume and increase cardiac output. In order to avoid injecting too much fluid in a short time, which may easily cause adverse reactions in elderly patients, such as pulmonary edema and heart failure, it is necessary to identify factors that can help to predict hypotension during anesthesia. The AUC of the IVCD max $_{\text {max }}$ in patients with hypotension indicated that it had the best predictive efficacy (Table 8, Figure 5A,B). The optimal threshold value of the $\mathrm{IVCD}_{\max }$ was 1.25 and $1.23 \mathrm{~cm}$ in the infusion group and the conventional group, respectively. The optimal thresholds 
were close, suggesting that with or without intravenous fluid, the IVCD criteria for predicting hypotension were similar. The results of this study are similar to the findings of Vieillard-Baron et al. (25), who reported that when the optimal threshold value was $\leq 1.3 \mathrm{~cm}$, the specificity of the $\mathrm{IVCD}_{\max }$ for predicting the volume response was $80 \%$. If the IVCD was measured by ultrasound before anesthesia in elderly patients who were treated with painless colonoscopy, hypotension was more likely to occur during anesthesia when the $\mathrm{IVCD}_{\max }$ was $<1.25$ or $<1.23 \mathrm{~cm}$. Ultrasoundguided individual intravenous infusion can reduce the risk of hypotension and has certain reference value.

Our study confirmed that appropriate intravenous infusion could shorten the post-anesthesia recovery time and improve the comfort of elderly patients who underwent painless colonoscopy. Additionally, measurement of the IVCD by ultrasound was an effective tool for assessing blood volume in these patients. Therefore, $\mathrm{IVCD}_{\max }$ could guide intravenous infusion and was a good predictor of hypotension. This might help guide the use of rehydration in patients undergoing painless colonoscopy, especially for the elderly. Ultrasound measurement of the volume of the inferior vena cava may also be a suitable and convenient non-invasive monitoring method, which could be used in clinical practice.

Certainly, this study has some limitations. Firstly, the sedation level was determined according to the response of the patients in this study. We recommend that bispectral index monitoring system is used to achieve the target sedation level in order to avoid the relative overdose of anesthetic drugs when hypotension occurs. Secondly, we did not compare electrolytes or renal function before and after bowel preparation. These comparisons can provide evidence to help explain one reason for prolonged time required for postoperative recovery. Thirdly, when hypotension occurred during anesthesia in patients who were in the left lateral position and whose intestine was in a state of inflation, the IVCD could not be accurately measured by ultrasound during procedure. Fourthly, if colloidal solution was selected as intravenous load, whether it could improve the degree of BP drop during anesthesia remains to be further explored. Finally, the optimal threshold of the IVCD for hypotension in elderly patients who undergo painless colonoscopy needs to be further investigated with a larger sample size and more protocol designs.

In conclusion, intravenous infusion promoted the rapid recovery of elderly patients who underwent painless colonoscopy, and ultrasonic measurement of the $\mathrm{IVCD}_{\max }$ helped to guide the intravenous infusion.

\section{Acknowledgments}

Funding: Science and Technology Project of Yuzhong District, Chongqing (20180137).

\section{Footnote}

Reporting Checklist: The authors have completed the STARD reporting checklist. Available at http://dx.doi.org/10.21037/ apm-20-2217

Data Sharing Statement: Available at http://dx.doi. org/10.21037/apm-20-2217

Conflicts of Interest: All authors have completed the ICMJE uniform disclosure form (available at http://dx.doi. org/10.21037/apm-20-2217). Dr. QX reports grants and personal fees from Science and Technology Project of Yuzhong District, Chongqing, during the conduct of the study. The other authors have no conflicts of interest to declare.

Ethical Statement: The authors are accountable for all aspects of the work in ensuring that questions related to the accuracy or integrity of any part of the work are appropriately investigated and resolved. All procedures performed in this study involving human participants were in accordance with the Declaration of Helsinki (as revised in 2013). The research plan was approved by the Medical Ethics Committee of West China Hospital of Sichuan University (No. 421, 2018). All patients or their guardians signed informed consent before the study.

Open Access Statement: This is an Open Access article distributed in accordance with the Creative Commons Attribution-NonCommercial-NoDerivs 4.0 International License (CC BY-NC-ND 4.0), which permits the noncommercial replication and distribution of the article with the strict proviso that no changes or edits are made and the original work is properly cited (including links to both the formal publication through the relevant DOI and the license). See: https://creativecommons.org/licenses/by-nc-nd/4.0/.

\section{References}

1. Goh KL, Quek KF, Yeo GT, et al. Colorectal cancer in Asians: A demographic and anatomic survey in Malaysian patients undergoing colonoscopy. Aliment Pharmacol Ther 2005;22:859-64. 
2. Morrison CE, Ritchie-Mclean S, Jha A, et al. Two hours too long: time to review fasting guidelines for clear fluids. Br J Anaesth 2020;124:363-6.

3. Labovitz AJ, Noble VE, Bierig M, et al. Focused cardiac ultrasound in the emergent setting: a consensus statement of the American Society of Echocardiography and American College of Emergency Physicians. J Am Soc Echocardiogr 2010;23:1225-30.

4. de Valk S, Olgers TJ, Holman M, et al. The caval index: an adequate non-invasive ultrasound parameter to predict fluid responsiveness in the emergency department? BMC Anesthesiol 2014;14:114-20.

5. Zhang Z, Xu X, Ye S, et al. Ultrasonographic measurement of the respiratory variation in the inferior vena cava diameter is predictive of fluid responsiveness in critically ill patients: systematic review and meta-analysis. Ultrasound Med Biol 2014;40:845-53.

6. Dipti A, Soucy Z, Surana A, et al. Role of inferior vena cava diameter in assessment of volume status: a metaanalysis. Am J Emerg Med 2012;30:1414-1419.e1.

7. Antonik LJ, Goldwater DR, Kilpatrick GJ, et al. A placeboand midazolam-controlled phase I single ascendingdose study evaluating the safety, pharmacokinetics, and pharmacodynamics of remimazolam (CNS 7056): Part I. Safety, efficacy, and basic pharmacokinetics. Anesth Analg 2012;115:274-83.

8. Steward DJ. A simplified scording system for the postoperative recovery room. Can Anaesth Soc J 1975;22:111-3.

9. Phillips NM, Street M, Kent B, et al. Post-anaesthetic discharge scoring criteria: Key findings from a systematic review. Int J Evid Based Healthc 2013;11:275-84.

10. Yogendran S, Asokumar B, Cheng DC, et al. A prospective randomized double-blinded study of the effect of intravenous fluid therapy on adverse outcomes on outpatient surgery. Anesth Analg 1995;80:682-6.

11. Xu T, Zheng J, An XH, et al. Norepinephrine intravenous prophylactic bolus versus rescue bolus to prevent and treat maternal hypotension after combined spinal and epidural anesthesia during cesarean delivery: a sequential dosefinding study. Ann Transl Med 2019;7:451.

12. Ayus JC, Levine R, Arieff AI. Fatal dysnatraemia caused by elective colonoscopy. BMJ 2003;326\:382-4.

13. Markowitz GS, Stokes MB, Radhakrishnan J, et al. Acute phosphate nephropathy following oral sodium phosphate bowel purgative: an underrecognized cause of chronic renal failure. J Am Soc Nephrol 2005;16\:3389-96.

14. Pierce JD, Shen QH, Thimmesch A, et al. The ongoing controversy: crystalloids versus colloids. J Infus Nurs 2016;39:40-4.
15. Holte K, Kehlet H. Compensatory fluid administration for preoperative dehydration-does it improve outcome? Acta Anaesthesiol Scand 2002;46:1089-93.

16. Bindels AJ, van der Hoeven JG, Graafland AD, et al. Relationships between volume and pressure measurements and stroke volume in critically ill patients. Crit Care 2000;4:193-9.

17. Mesquida J, Gruartmoner G, Ferrer R, et al. Passive leg raising for assessment of volume responsiveness: a review. Curr Opin Crit Care 2017;23:237-43.

18. Monnet X, Teboul JL. Assessment of volume responsiveness during mechanical ventilation: recent advances. Crit Care 2013;17:217.

19. Ferrada P, Anand RJ, Whelan J, et al. Qualitative assessment of the inferior vena: useful tool for the evaluation of fluid status in critically ill patients. Am Surg 2012;78:468-70.

20. Laher AE, Watermeyer MJ, Buchanan SK, et al. A review of hemodynamic monitoring techniques, methods and devices for the emergency physician. Am J Emerg Med 2017;35:1335-47.

21. Wo CC, Shoemaker WC, Appel PL, et al. Unreliability of blood pressure and heart rate to evaluate cardiac output in emergency resuscitation and critical illness. Crit Care Med 1993;21:218-23.

22. Kanji HD, McCallum J, Sirounis D, et al. Limited echocardiography-guided therapy in subacute shock is associated with change in management and improved outcomes. J Crit Care 2014;29:700-5.

23. Hirsch J, DePalma G, Tsai TT, et al. Impact of intraoperative hypotension and blood pressure fluctuations on early postoperative delirium after non-cardiac surgery. Br J Anaesth 2015;115:418-26.

24. Reich DL, Hossain S, Krol M, et al. Predictors of hypotension after induction of general anesthesia. Anesth Analg 2005;101:622-8.

25. Vieillard-Baron A, Evrard B, Repesse X, et al. Limited value of end-expiratory inferior vena cava diameter to predict fluid responsiveness impact of intra-abdominal pressure. Intensive Care Med 2018;44:197-203.

Cite this article as: $\mathrm{Xu} \mathrm{Q}$, Tu H, Xiang S, Tan Q, Wang X. The effect of intravenous infusion on the rapid recovery of elderly patients treated with painless colonoscopy and the value of ultrasonic measurement of the inferior vena cava diameter in guiding intravenous infusion. Ann Palliat Med 2021;10(1):6173. doi: 10.21037/apm-20-2217 